\title{
Persistent Conductivity in TPyP:TSPP Organic Nanorods Induced by Ion Bombardment
}

\author{
Marshall van Zijll, Bryan Borders, Ursula Mazur, K.W. Hipps \\ Department of Chemistry and Materials Science and Engineering Program, \\ Washington State University, Pullman, WA, 99164-4630, USA
}

(Dated: May, 8, 2016)

\section{SAMPLE PREPARATION}

The interdigitated electrode (IDE) consists of a $20 \mathrm{~nm}$ thick layer of Au deposited onto a glass slide: the size of the interdigitated section, located in the center of Fig. 1a, is $127 \times 127 \mu \mathrm{m}^{2}$ and consists of 34 interdigitated rows separated by $500 \mathrm{~nm}$ gaps. Following self-assembly of the nanorods in solution, small drops of solution were deposited onto the IDE and allowed to evaporate, leaving a deposition of randomly oriented nanorods (Figs. 1b and 1e). Depending on the concentration of rods in the drop of solution, typically 100 's-1000's of rods were deposited onto the interdigitated section, a good portion of which appeared to bridge the anode rows to the cathode rows. For some samples we scraped away the interdigitated section (Fig. 1d) leaving a $1 \mathrm{~mm}$ wide gap, this large gap required measureable conduction to travel from rod to rod.

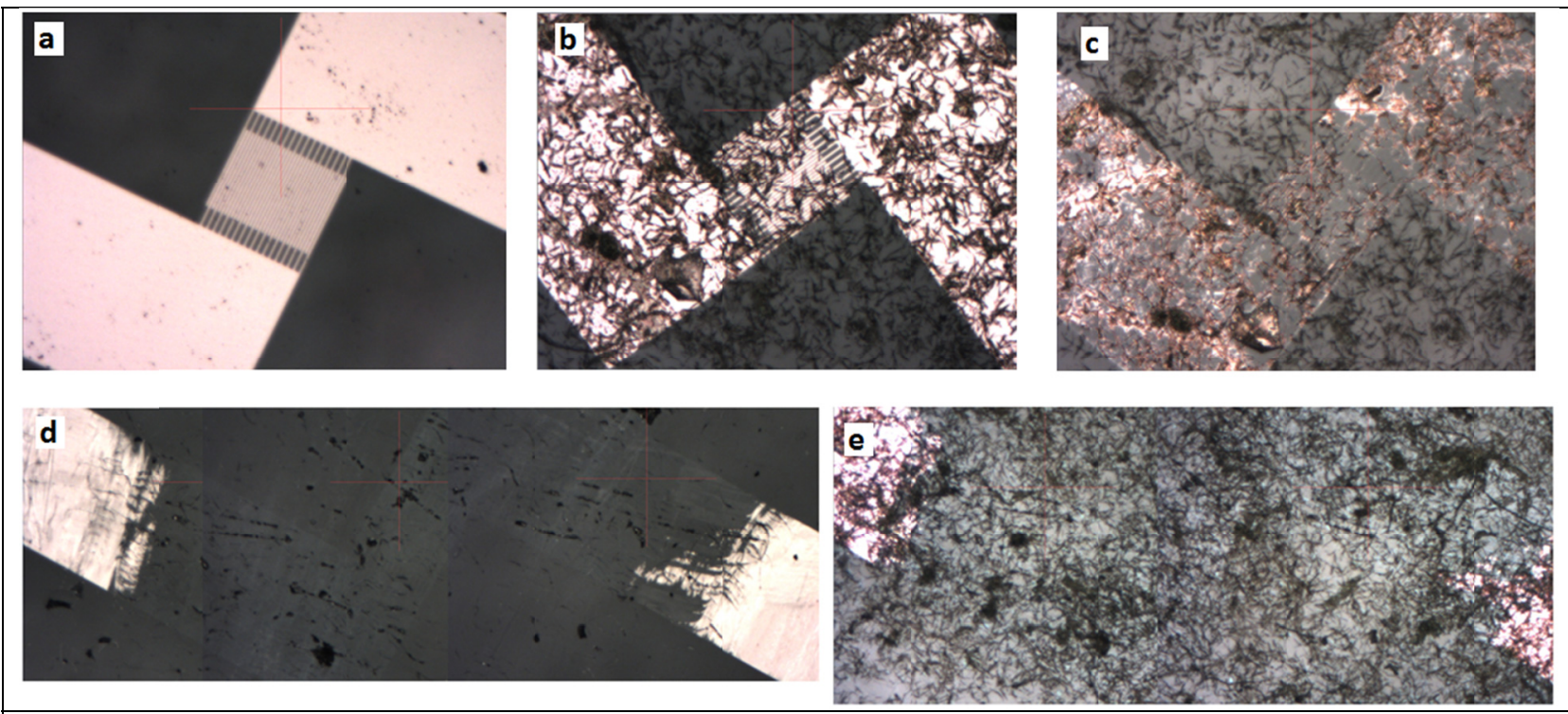

Figure S1. Optical images of a) a clean IDE, b) (a) deposited with nanorods, c) (b) following $300 \mathrm{~s}$ of ion bombardment with $1 \mathrm{keV}$ and $5 \times 10^{-6}$ torr $\mathrm{Ar}^{+}$(experiment 8a in Table 2), d) IDE with $1 \mathrm{~mm}$ gap created by scraping away gold, e) (d) deposited with nanorods. The gold located directly beneath deposited nanorods was protected by the nanorods from being sputtered away. Heavy depositions of nanorods prevented sputtering of the electrodes.

\section{CONTROL EXPERIMENTS}

Ion bombardment of our sample holder without an IDE reveals the effects of charging as seen in Fig. 2a. Charging of the sample holder introduces a large initial current which decays to a few pAs within about $50 \mathrm{~s}$ of continuous bombardment, after ion bombardment ceases the charging dissipates in about $20 \mathrm{~s}$. 


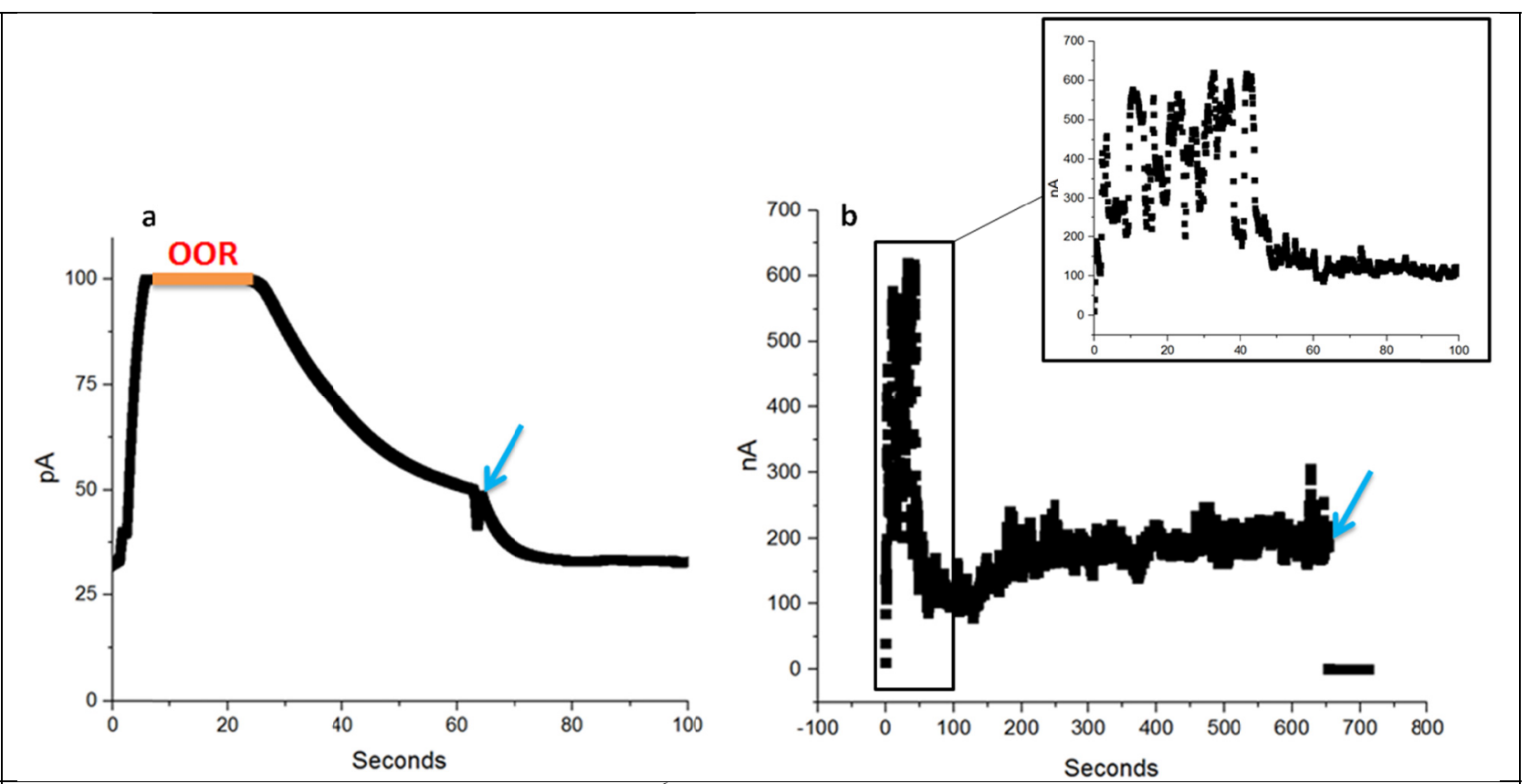

Figure S2. Ion bombardment with $500 \mathrm{eV}, 1.5 \times 10^{-6} \mathrm{Ar}^{+}$performed on a) a sample holder without an IDE, b) an IDE with no nanorods deposited. Sputtering begins at $0 \mathrm{~s}$ in both cases, and ceases at the time indicated by the blue arrow.

Ion bombardment of a blank IDE (Fig. 2b) allowed us to observe the effects due to both charging and conductance through the Ar plasma. We see an initial large jump in current due to charging which lasts about $50 \mathrm{~s}$ (inset in Fig. $2 b)$. The current subsequently approaches a somewhat-constant current. The current quickly dropped to 0 when ionbombardment ceased. Due to the effects of charging and plasma conduction, the current during ion-bombardment cannot be directly measured with smaller currents $(\leq 1 \mu \mathrm{A})$, and for larger currents we observe a large instability in the current during the first $50 \mathrm{~s}$, as well as an offset on the order of $0.1-1 \mu \mathrm{A}$ during the entirety of the measurement.

\section{VARIOUS EFFECTS OF ION BOMBARDMENT}

The large kinetic energy of incident ions, and also the sputtering arising from ion-bombardment, suggest possibilities as to how ion-bombardment may increase the conductivity of these nanorods in a manner fundamentally different from PPC. In particular:

- Ar embedment

- Co-sputtering from the substrate

- Preferential sputtering of rods

Calculations performed using "the Stopping and Range of Ions in Matter" (SRIM) software are shown in table I for TPyP:TSPP nanorods and IDE components. SRIM predicts the range and straggle of incident Ar+ impacting the nanorods to be $35 \AA$ and $10 \AA$. These values are shallow due to the low energy of ion bombardment. Using values calculated with SRIM (Table I), the time to sputter away all the carbon in the deposited TPyP:TSPP nanorods is estimated to be $10,000-100,000 \mathrm{~s}$ at $500 \mathrm{eV}$, depending on the amount of deposition. Typical exposure times in our experiments were 100's - 1,000's of seconds. Consistent with our calculations, we observed that even when much of the gold was noticeably sputtered, the rods did not show significant degradation. The gold located directly beneath deposited nanorods was protected by the nanorods from being sputtered away. Heavy depositions of nanorods prevented sputtering of the electrodes. 
Table I: SRIM calculations of $\mathrm{Ar}^{+}$bombardment at $500 \mathrm{eV}$.

\begin{tabular}{|l|l|l|}
\hline & $\begin{array}{l}\text { Sputtering Yield (Atoms } \\
\text { per incident ion) }\end{array}$ & $\begin{array}{l}\text { Ar longitudinal } \\
\text { range/straggle }\end{array}$ \\
\hline TSPP:TPyP (1000 A) & $\begin{array}{l}(\mathrm{C}, \mathrm{H}, \mathrm{N}, \mathrm{O}, \mathrm{S}) \\
(0.040,0.086,0.030, \\
0.030,0.007)\end{array}$ & $35 \mathrm{~A} / 10 \mathrm{~A}$ \\
\hline Au (200 A) / Silica (1000 A) & 2.46 & $14 \mathrm{~A} / 10 \mathrm{~A}$ \\
\hline Silica (1000 A) & $\begin{array}{l}(\mathrm{Si}, \mathrm{O}) \\
(0.112,0.489)\end{array}$ & $31 \mathrm{~A} / 11 \mathrm{~A}$ \\
\hline
\end{tabular}

\section{Ar embedment}

If $\mathrm{Ar}^{+}$embedment were playing a role to increase conductivity, it could have an affect similar to doping the system. Typical doping would change dark conductivity rather than persistent conductivity, but our experiments suggest a small limit on the dark conductivity.

\section{Co-sputtering}

Experiments were performed with nanorods deposited onto non-conductive substrates (Fig. 1d), and the persistent conductivity was similar to experiments performed on conductive substrates. This certainly eliminates co-sputtering as a possibility.

\section{Preferential sputtering}

Ion bombardment of the surface could cause preferential sputtering of particular elements which may result in a conductive carbon skeleton being left behind. We do not suspect that preferential sputtering of the rod is the cause of increasing conductivity. Similar to ion-embedment and co-sputtering, preferential sputtering would likely cause an change in the dark conductivity, which is something we do not observe.

\section{STM IMAGES OF ION BOMBARDED NANORODS}

Nanorods were deposited onto both gold/mica and graphite substrates, and imaged using STM. Images were initially attempted during photoillumination, but distinguishable nanorods were not image-able. STM images revealed a substrate contaminated with unintegrated porphyrins. During scanning of a single cross-section, a rod would sometimes appear, but subsequent passes of the scanning tip would sweep the rod away. Nanorods were successfully imaged following 100-300 s ion bombardment with typical parameters. By performing ion bombardment, nonintegrated porphyrins were sputtered away, and conductivity in the nanorods was induced. After a particular ionbombardment experiment the persistent conductivity was allowed to decay for 3 months and then STM work was performed. During 4 hours of imaging only 4 rods were located. This sample was then illuminated with the $445 \mathrm{~nm}$ laser and again imaged. During this imaging, 21 rods were located in 1 hour. These observations suggest that the initial failure to image rods during photoillumination was due at least in part to contamination of the surface.

Figures 3-7 are STM images of TPyP:TSPP nanorods on a gold/mica substrate. They are shown here to give an indication of their appearance following ion bombardment. Sputtering due to ion bombardment is responsible for the emphasized appearance of repetitive lines and grid-like patterns on the nanorods, these features are likely associated with the rod's crystallinity. 

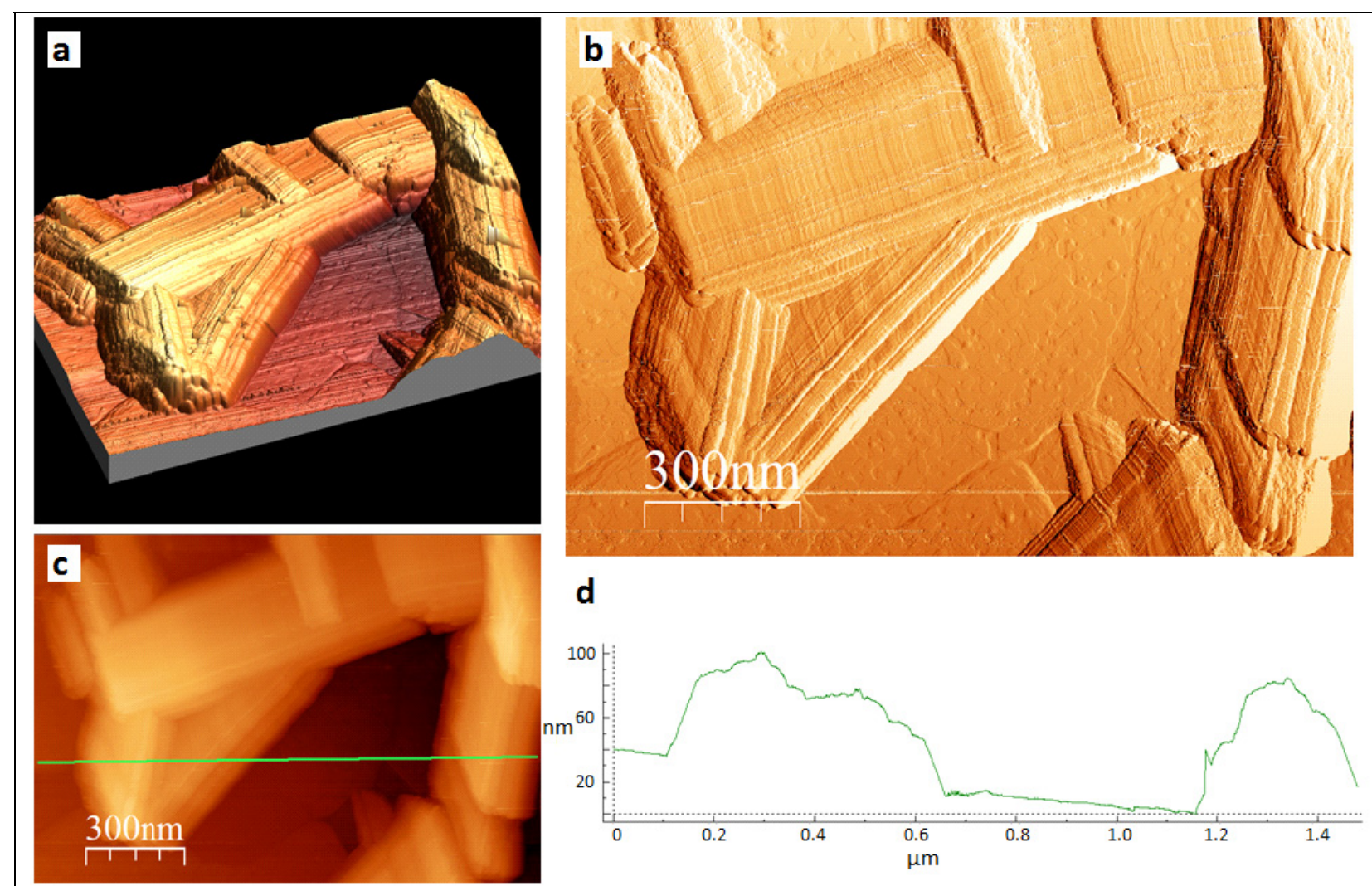

Figure S3. STM image of nanorods on Au on Mica, $40 \mathrm{pA}, 2.0 \mathrm{~V}$ sample. This sample was ion bombarded for $300 \mathrm{~s}$ with $1 \mathrm{keV}$ and $5 \times 10^{-6}$ torr $\mathrm{Ar}^{+}$.
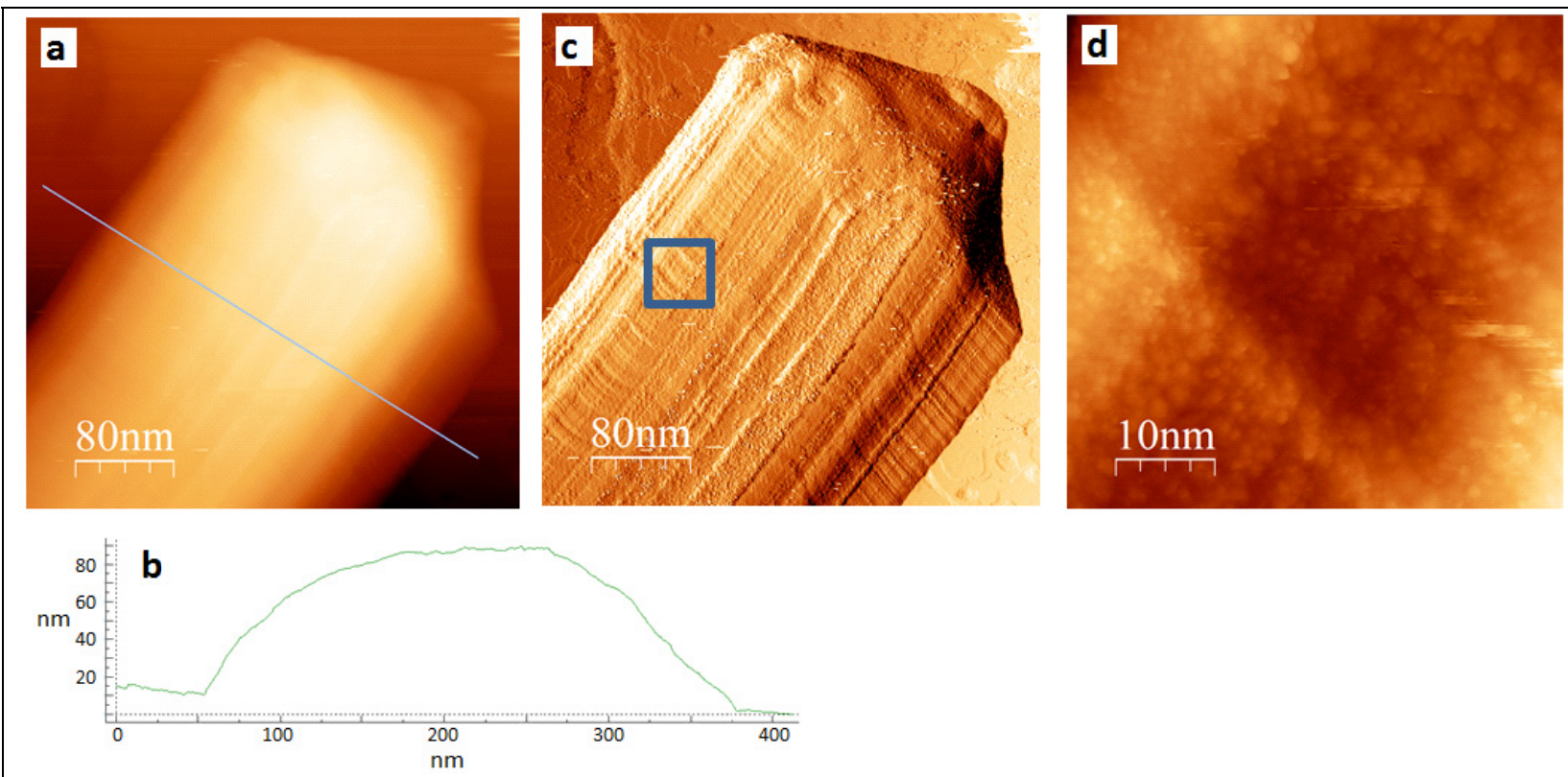

Figure S4. STM images of a nanorod on Au/Mica, $40 \mathrm{pA},-2.0 \mathrm{~V}$ sample. This sample was ion bombarded for $300 \mathrm{~s}$ with $1 \mathrm{keV}$ and $5 \times 10^{-6}$ torr $\mathrm{Ar}^{+}$. a) topographical with line profile (b), c) derivative of (a), d) zoomed in topographical image of location indicated in (c). 


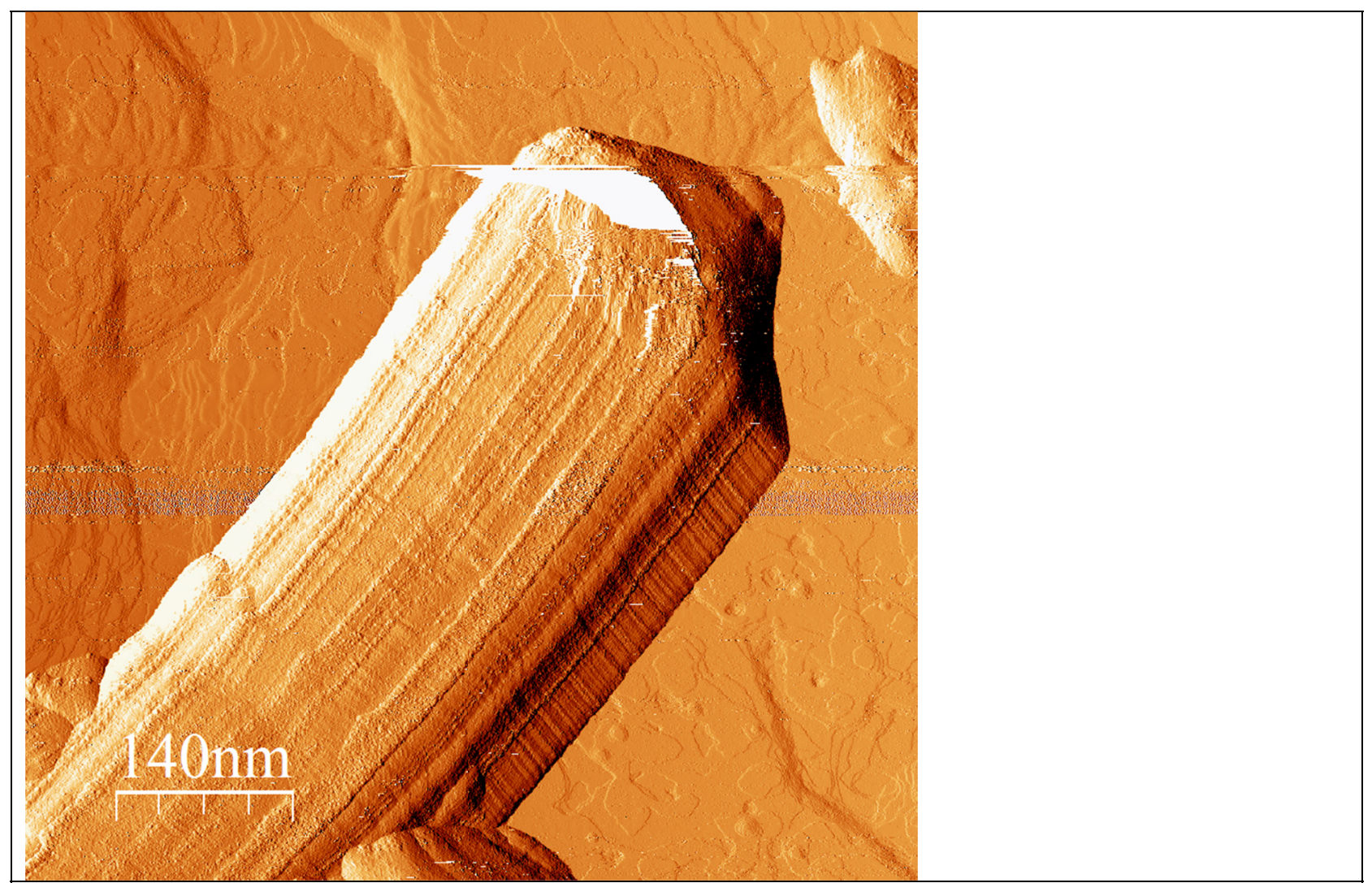

Figure S5. Larger STM image of nanorod shown in Fig. 4, 40 pA, -2.0 V sample. This sample was ion bombarded for $300 \mathrm{~s}$ with $1 \mathrm{keV}$ and $5 \times 10^{-6}$ torr $\mathrm{Ar}^{+}$. 


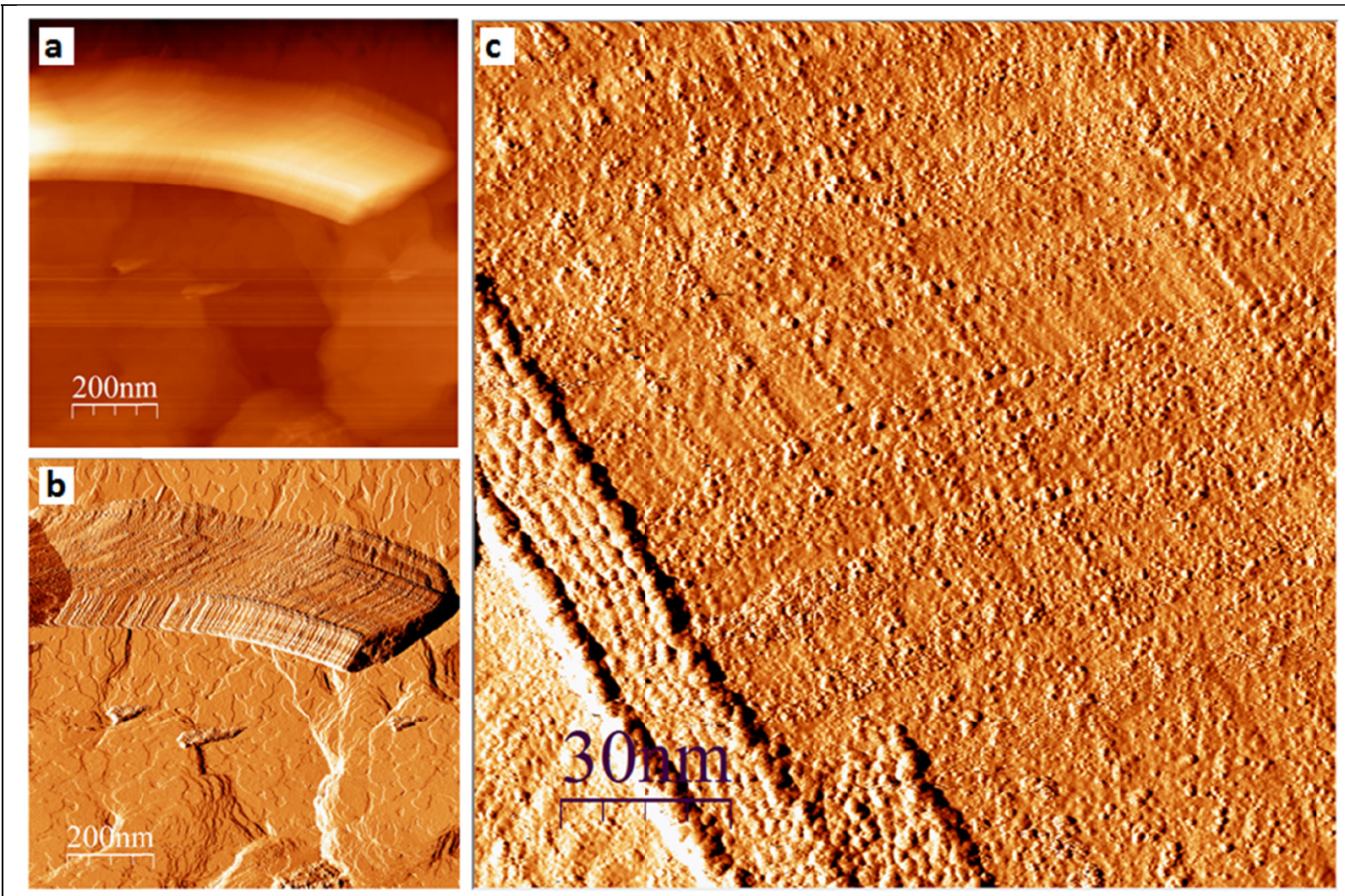

Figure S6. STM image of a nanorod on Au on Mica, $40 \mathrm{pA}, 1.5 \mathrm{~V}$ sample. This sample was ion bombarded for $60 \mathrm{~s}$ with $1 \mathrm{keV}$ and $5 \times 10^{-6}$ torr $\mathrm{Ar}^{+}$. b) is a derivative image of a). c) is a zoomed-in derivative image of a section of a). The exact location is unsure due to the drift during the larger-area image.
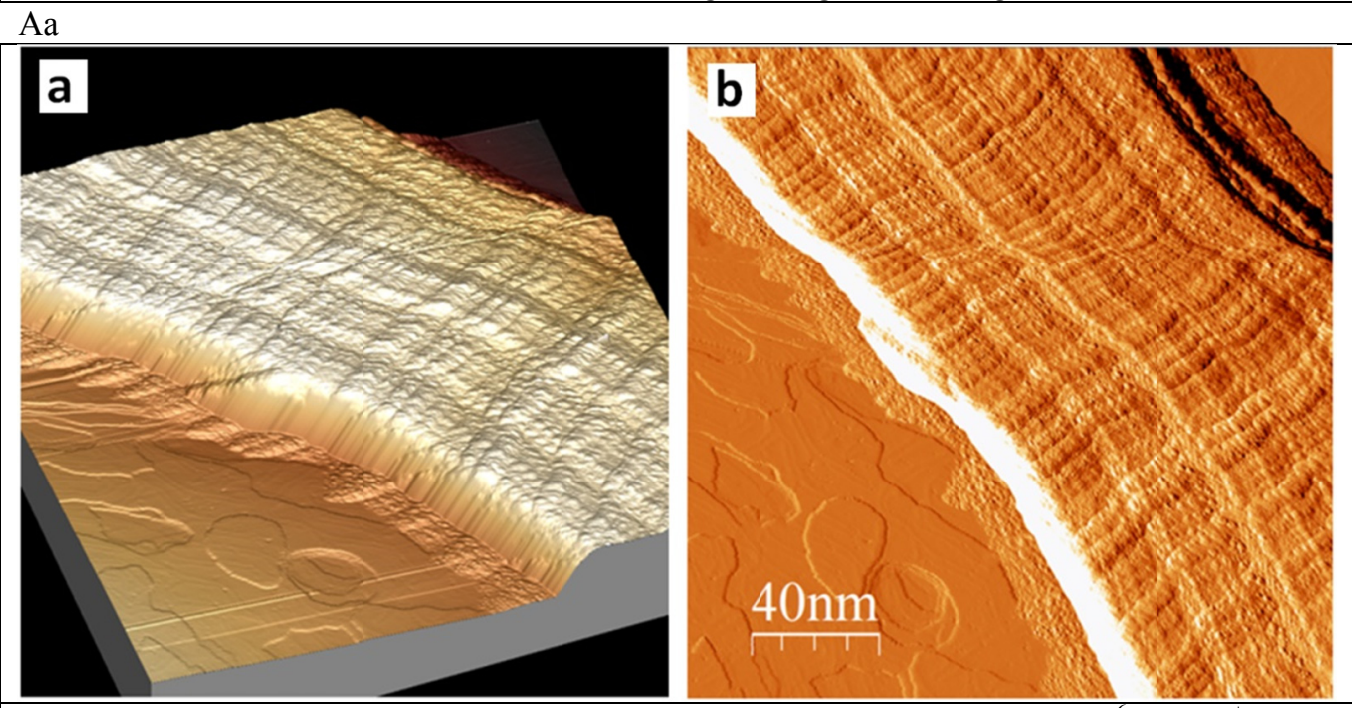

Figure S7. STM images of a TPyP:TSPP nanorod following $500 \mathrm{eV}$ and $5 \times 10^{-6}$ torr $\mathrm{Ar}^{+}$ion bombardment for 240 $\mathrm{s}$ at $500 \mathrm{eV}$ and $5 \times 10^{-6}$ torr $\mathrm{Ar}^{+}$. a) a $3 \mathrm{D}$ representation, b) a derivative image. 
Table II show parameters associated with best fits for decay curves of persistent conductivity resulting from ion bombardment. The decay curves were fit with a stretched-exponential function:

$$
. I=I_{0} * \exp \left(-\left(\frac{t}{\tau}\right)^{\beta}\right),(0<\beta \leq 1)
$$

Eight of the experiments listed in table II were continuously recorded for between 24 and 90 hours following ion bombardment.

Table II. Experiment best-fit components of the stretched exponential for decay curves.

\begin{tabular}{|c|c|c|c|c|c|c|c|}
\hline $\begin{array}{c}\text { Sample. } \\
\text { Experiment }\end{array}$ & $\mathbf{1 , a}$ & $\mathbf{1 , b}$ & $\mathbf{2 , a}$ & $\mathbf{2 , b}$ & $\mathbf{2 , c}$ & $\mathbf{3 , a}$ & $\mathbf{3 , ~ b}$ \\
\hline$\beta$ & 0.16 & 0.16 & 0.135 & 0.117 & 0.1 & 0.147 & 0.15 \\
\hline$\tau$ & $1300 \mathrm{~s}$ & $2800 \mathrm{~s}$ & $1000 \mathrm{~s}$ & $1000 \mathrm{~s}$ & $3000 \mathrm{~s}$ & $1500 \mathrm{~s}$ & $1500 \mathrm{~s}$ \\
\hline$I_{0}$ & $76 \mathrm{pA}$ & $1.3 \mathrm{nA}$ & $160 \mathrm{pA}$ & $530 \mathrm{pA}$ & $29 \mu \mathrm{A}$ & $320 \mathrm{pA}$ & $220 \mathrm{pA}$ \\
\hline
\end{tabular}

Table II cont.

\begin{tabular}{|c|c|c|c|c|c|c|c|}
\hline $\begin{array}{c}\text { Sample, } \\
\text { Experiment }\end{array}$ & $\mathbf{3 , c}$ & $\mathbf{3 , d}$ & $\mathbf{4 , a}$ & $\mathbf{4 , b}$ & $\mathbf{5 , a}$ & $\mathbf{6 , a}$ & $\mathbf{7 , a}$ \\
\hline$\beta$ & 0.12 & 0.106 & 0.13 & 0.138 & 0.1 & 0.16 & 0.105 \\
\hline$\tau$ & $500 \mathrm{~s}$ & $2500 \mathrm{~s}$ & $1000 \mathrm{~s}$ & $500 \mathrm{~s}$ & $1000 \mathrm{~s}$ & $500 \mathrm{~s}$ & $2000 \mathrm{~s}$ \\
\hline$I_{0}$ & $260 \mathrm{nA}$ & $5.4 \mu \mathrm{A}$ & $0.94 \mathrm{nA}$ & $5.2 \mathrm{nA}$ & $100 \mathrm{nA}$ & $22 \mu \mathrm{A}$ & $27 \mu \mathrm{A}$ \\
\hline
\end{tabular}

Figure $8 \mathrm{a}$ is identical to Fig. $4 \mathrm{c}$ in the primary article. Figures $8 \mathrm{~b}-\mathrm{d}$ were obtained by similar experiments on different samples. The experimental details can be found in the table with Fig. 8.

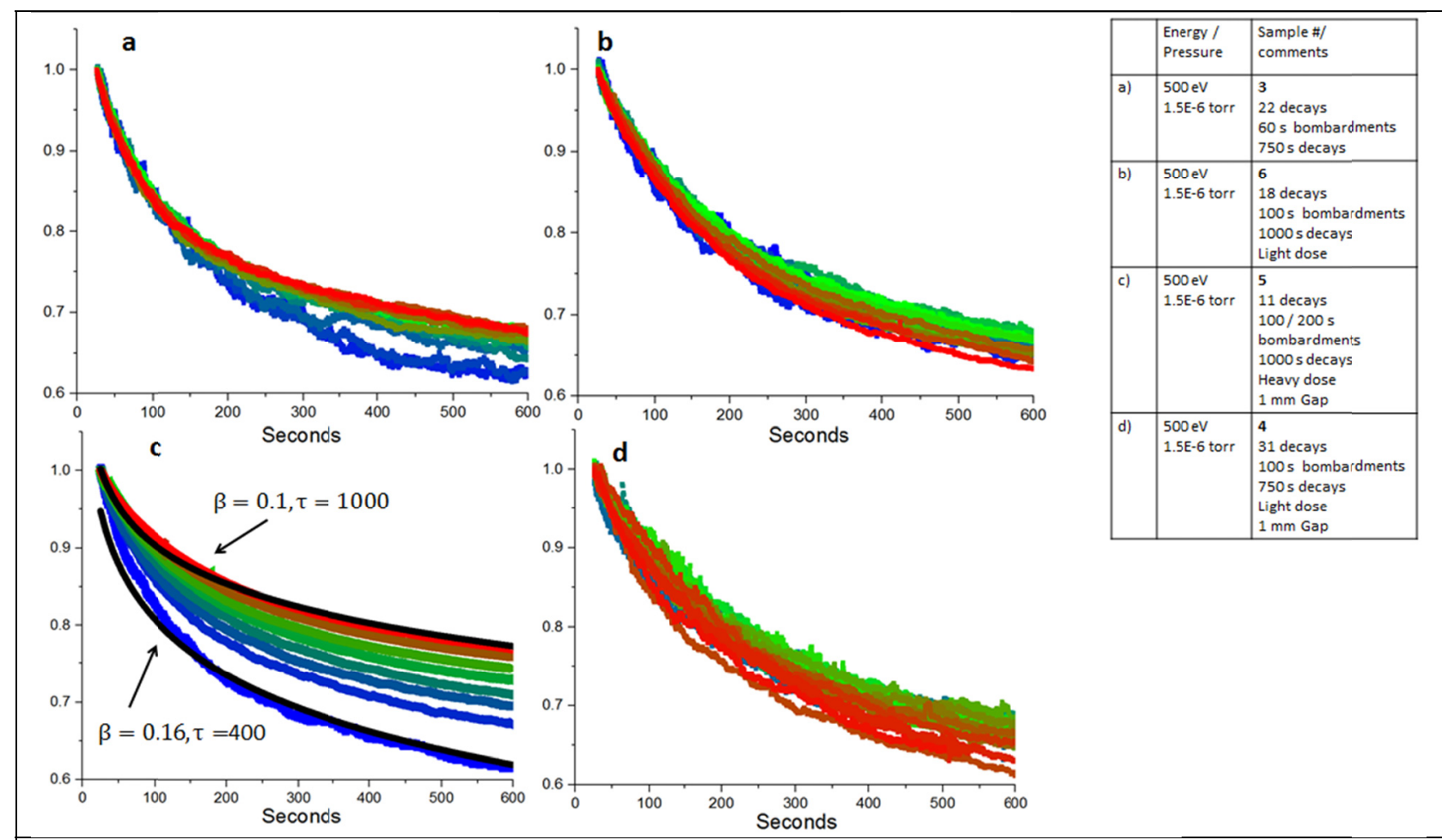

Figure S8. a-d) show normalized decays in persistent conductivity from data collected by many subsequent cycles of ion bombardment and relaxation. Earlier decays are blue and later decays are green then red. The table shows the associated ion bombardment parameters, sample number corresponding to Table II, and comments. 
Figure $9 \mathrm{a}$ is identical to Figs $4 \mathrm{~b}$ in the primary article. Figures $9 \mathrm{~b}-\mathrm{d}$ were obtained by similar experiments on different samples. The experimental details can be found in the table with Fig. 9. Figure 9e and f show growth curves which were obtained by direct measurement, possible because of the higher conductivities. Figure $9 \mathrm{~g}$ contains curves labeled " $a$ " and " $b$ ", these are identical to curves labeled " $a$ " and " $c$ " in Fig. 6a of the primary article, but here they are shown overlaid to allow comparison of the slopes.

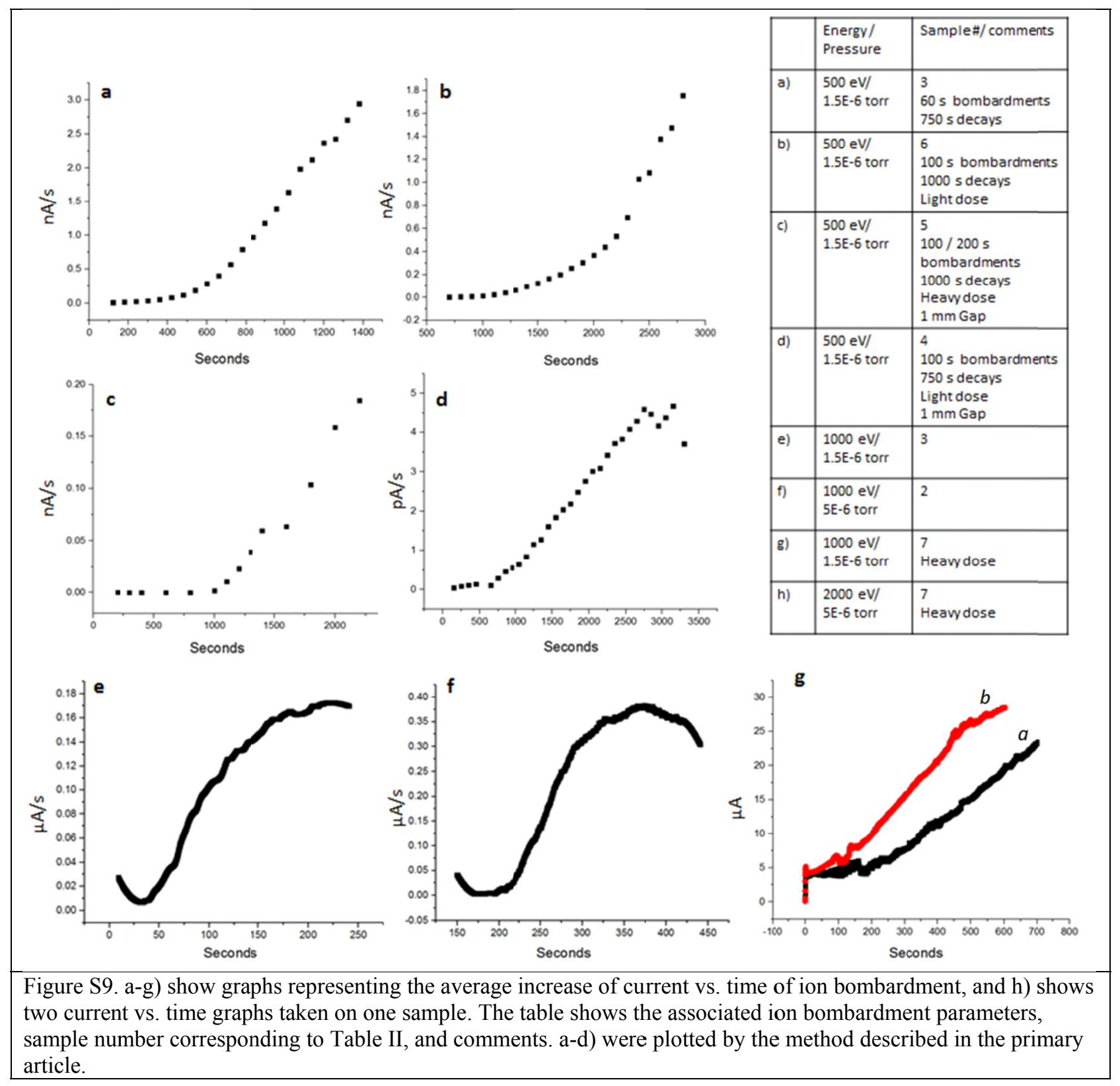

Figure 10a shows the current measured during a repetition of nine different $60-80 \mathrm{~s}$ ion bombardments each followed by 1000-1500 s decays. The colored decays in Fig. 10a are sampled at $0.1 \mathrm{~Hz}$ in order to more easily observe the curvature during bombardment. The measurements during ion bombardment are shown in black, and the decaying persistence curves are colored. The ion bombardment exposures labeled i-iii in Fig. 10a all cause an increase in persistence as seen by comparing their decay curves. The ion bombardment exposures labeled iv-ix each cause the persistence to decrease to values lower than the previous decay curves were predicted to fall. The flux and energy of each particular bombardment are listed in the tables in Fig. 10. 


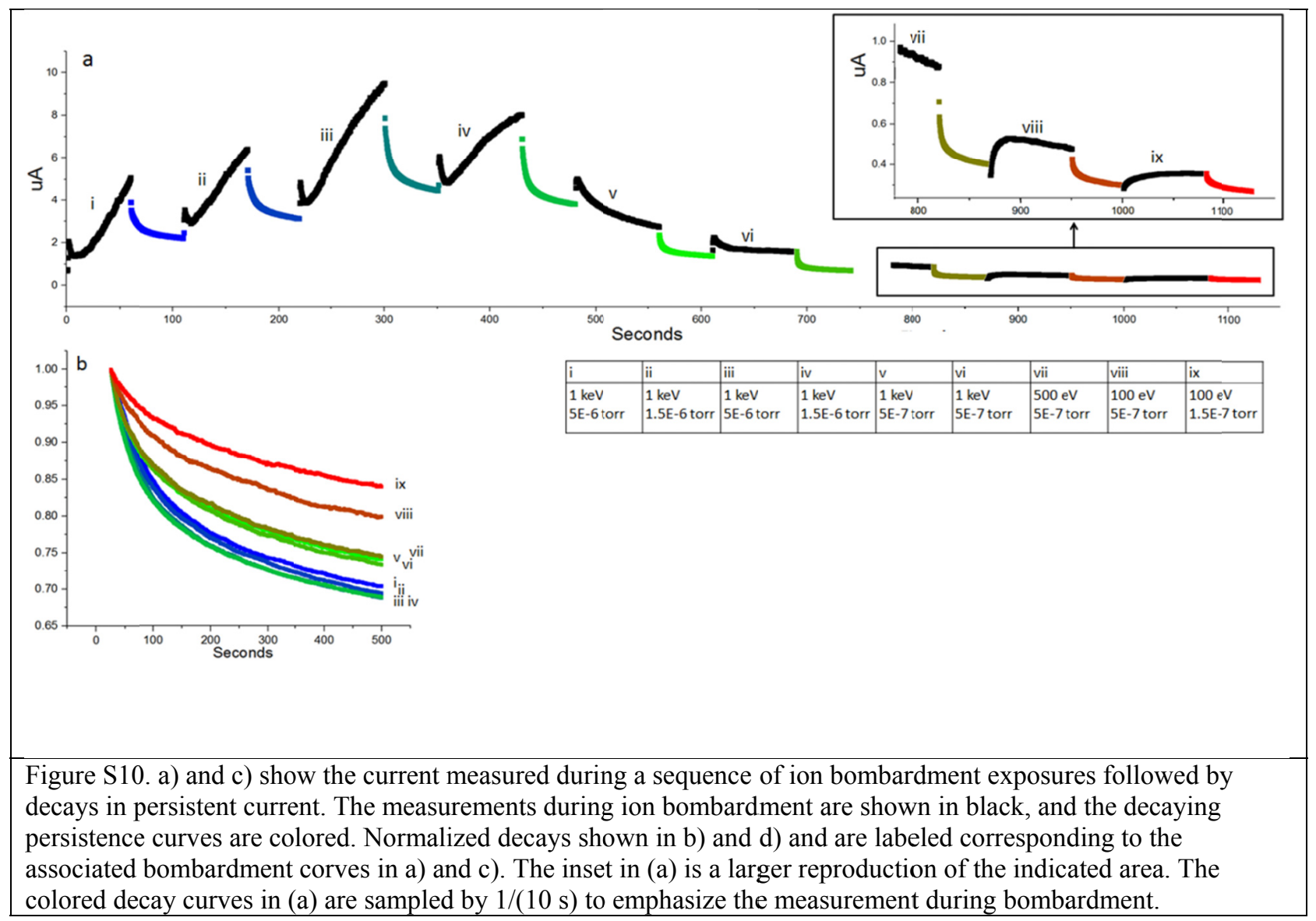

\section{NUMERICAL CALCULATION CODE}

\section{Numerical Calculation written in $\mathrm{C}++$ :}

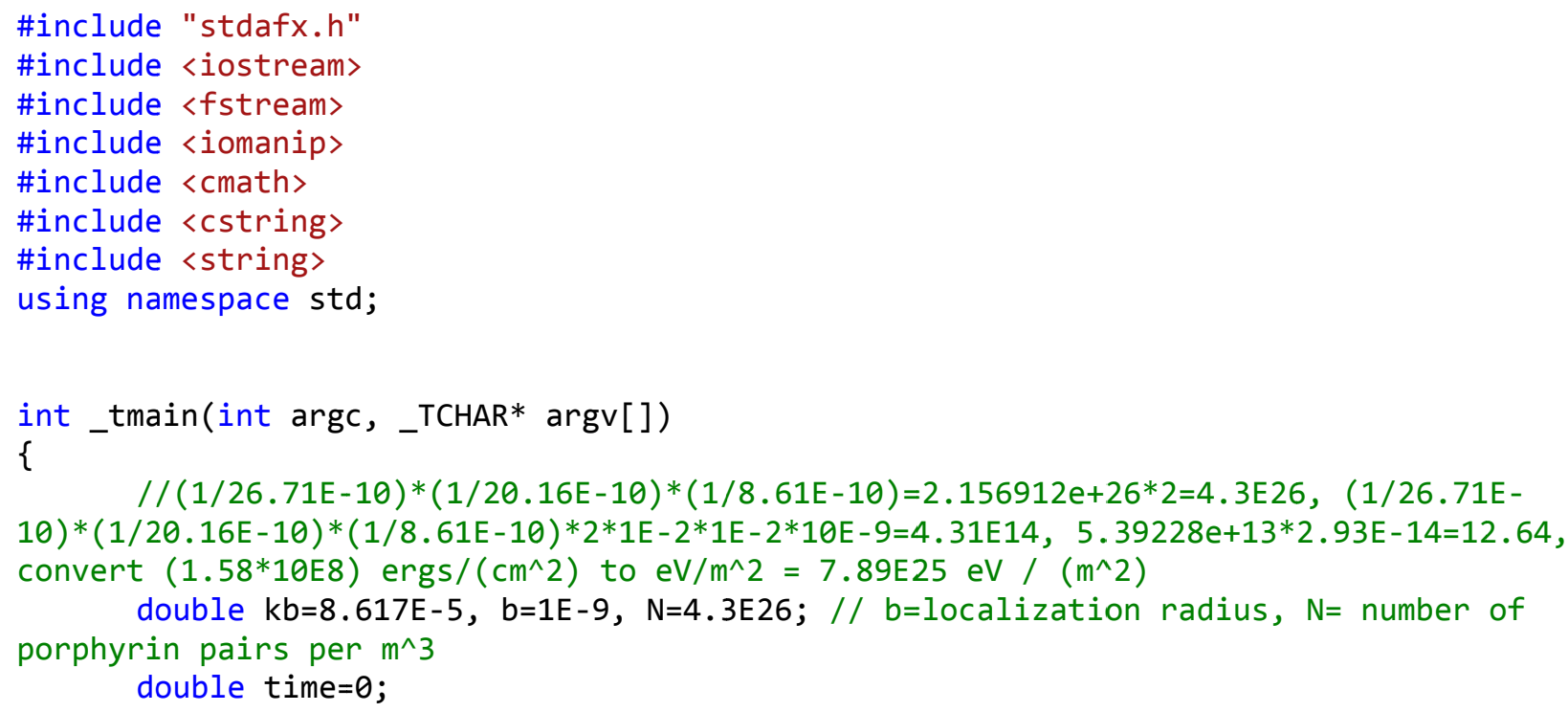


2E18;

double energyPerSecond $=2 \mathrm{E} 19, \mathrm{Ee}=1.33, \mathrm{Er}=1.13, \mathrm{ke}=1 \mathrm{E} 13, \mathrm{Cnr}=7.9 \mathrm{E} 25, \mathrm{knr}=$ beamTime $=120$;

double timeIntervalDefault $=1 \mathrm{E}-5$, Tbath $=300$, Tsubstrate $=300$, maxTime $=2460$, double tempSlices[1002], thetaSlices[1000], dummyTempSlices[1001],

dummyThetaSlices[1000], temperatureArray[30000][10], thetaArray[30000][10],

thetaBackup[100], timeArray[30000], IArray[30000];

int omegaCount $=0$, arraySize $=0$;

// if Er too low,

double $\mathrm{Ne}, \mathrm{Nr}, \mathrm{Kn}$, aDistance, $\mathrm{kr}=\mathrm{ke}$, vij, ue, Csurface $=\mathrm{Cnr} * 1 \mathrm{E}-8, /{ }^{*} 10 \mathrm{~nm}$

deep*/ slices=50, dxnr=1E-6, ksubstrate=1E16/5, /*E19*/ Csubstrate=1.389E23/5; //

$\mathrm{m}^{\wedge} 2$ sample, nanorods are 1 um deep, substrate is $2 \mathrm{~mm}$ deep

$/ /$ csurface $=e V /\left(m^{\wedge} 2 * 10 n m * K\right), \quad C n r=e V /\left(m^{\wedge} 3 * K\right), \quad k n r=e V /(m * s * K)$,

ksubstreate $=e V /(2 m m * s * K), d x, d x 2=m$

double OmegaInitial $=k e * \exp (-1.0 * \mathrm{Ee} /(\mathrm{kb} *$ Tsubstrate $)) /(\mathrm{ke} * \exp (-$

$1.0 * \mathrm{Ee} /(\mathrm{kb} * \mathrm{~T}$ substrate $))+\mathrm{kr} * \exp (-1.0 * \mathrm{Er} /(\mathrm{kb} *$ Tsubstrate $)))$; //must initialize Omega . double prevCapture $=-1 * \max T$ ime $/ 30000.0$, prevIteration $=-1$, timeInterval $=$ timeIntervalDefault, anotherCounter $=0 ; / /$ counters

//counters int iterationCount $=0$, iterationCountReal $=0$, iterationCountRealPrev $=0$;

double timeGap = maxTime $/ 30000.0 ; / /$ gap between array entries

bool restart $=$ false, beamon=true;

for(int $i=0$; $i<s l i c e s ; i++) / /$ initialize arrays

\{

tempSlices $[i]=300$;

\}

thetaSlices [i]=OmegaInitial;

while $($ time $<\operatorname{maxTime})$

\{

iterationCount++;

if(iterationCount != previteration) //if it has to redo iteration more

slowly, iterationcount $==$ previteration. This is called when it moves onto next

iteration successfully to speed it up.

\{

timeInterval = timeIntervalDefault;

\}

previteration = iterationCount;

double thisEnergy = energyPerSecond*timeInterval;

energy

if(anotherCounter > beamTime) //turns on and off the beam, and changes

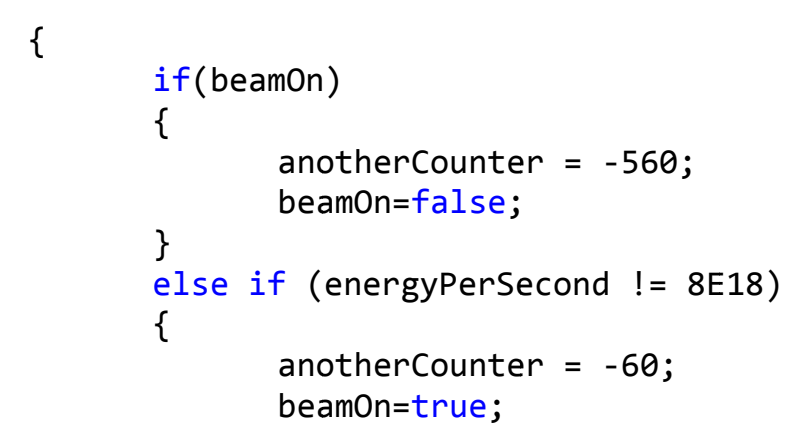




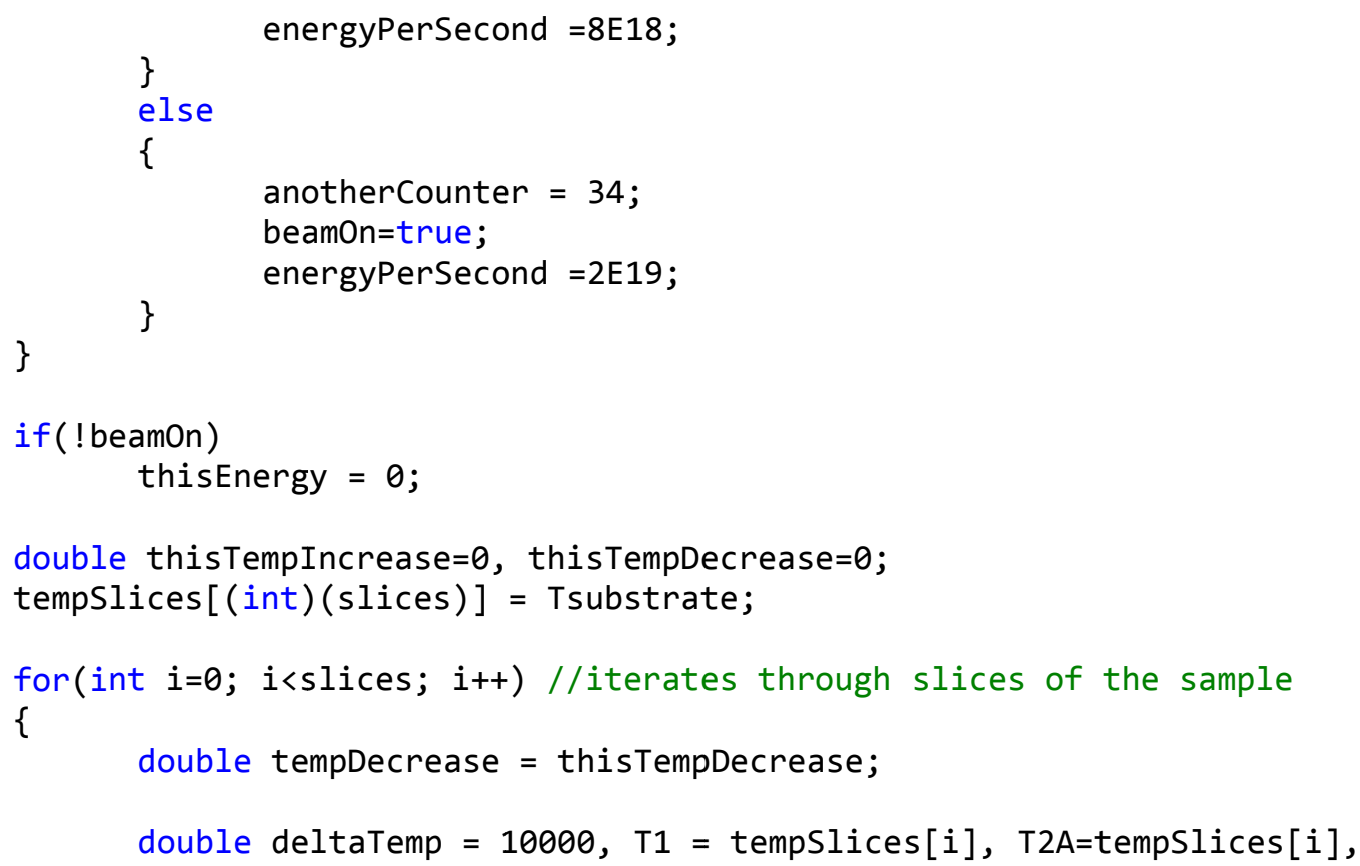
and final temperatures $\mathrm{T} 2 \mathrm{~A}$ and $\mathrm{T} 2 \mathrm{~B}$.

//This loop continues until deltaTemp=T2A-T2B $<=0.0001$

while(abs(deltaTemp) > 0.0001) //set temperature change precision

per iteration

$$
\{
$$

iterationCountReal++;

NeEst $=0$; NrEst $=0$;

double jlength $=4$;

for(int $j=0 ; j<j l e n g t h ; j++) / /$ considering the initial and

final temperature ( $\mathrm{T} 1$ and $\mathrm{T} 2 \mathrm{~A}$ ), estimates the number of sites excited ( $\mathrm{Ne}$ ) or relaxed $(\mathrm{Nr})$ in this slice

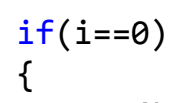


if(NrEst > N*1E-8*thetaSlices[i])

NrEst $=\mathrm{N} * 1 \mathrm{E}-8 *$ thetaSlices $[\mathrm{i}]$;

if (NeEst > N*1E-8*(1-thetaSlices[i]))

NeEst $=\mathrm{N} * 1 \mathrm{E}-8^{*}(1-$ thetaSlices $[\mathrm{i}])$;

\}

else // avoids over excitation/relaxation

\{

if(NrEst > $\mathrm{N}^{*} \mathrm{dxnr} /($ slices -1$) *$ thetaSlices [i] $)$

NrEst $=\mathrm{N} * \mathrm{dxnr} /($ slices -1$) *$ thetaSlices $[\mathrm{i}]$;

if $\left(\right.$ NeEst $>\mathrm{N}^{*} \mathrm{dxnr} /($ slices -1$) *(1$-thetaSlices $\left.[\mathrm{i}])\right)$

\}

NeEst $=\mathrm{N} * \mathrm{dxnr} /($ slices -1$) *(1-$ thetaSlices $[\mathrm{i}])$;

energyChangeEst $=($ NeEst $-\mathrm{NrEst}) *($ Ee-Er $)$;

//considering the initial and final temperature (T1 and

$T 2 B)$, estimates the temperature change in this slice

if $(i==\theta)$

\{

thisTempIncrease $=$ thisEnergy $/(\mathrm{Cnr} * 1 \mathrm{E}-8)$ -

energyChangeEst/(Cnr*1E-8); // im keeping the first layer at 10nm, penetration depth thisTempDecrease $=$

timeInterval*knr*((tempSlices[i])-tempSlices[i+1])/(Cnr*1E-8);

\}

else if $(i==1)$

\{

thisTempIncrease $=$ tempDecrease $*(\mathrm{Cnr} * 1 \mathrm{E}-$

8)/(Cnr*dxnr/(slices-1))-energyChangeEst/(Cnr*dxnr/(slices-1));

thisTempDecrease $=$

timeInterval*knr*((tempSlices[i])-tempSlices[i+1])/(Cnr*dxnr/(slices-1));

\}

else if $(i==$ slices -1$)$

\{

thisTempIncrease $=$ tempDecrease-

energyChangeEst/(Cnr*dxnr/(slices-1));

thisTempDecrease $=$

timeInterval*knr*((tempSlices[i])-Tsubstrate)/(Cnr*dxnr/(slices-1));

\}

else

\{

energyChangeEst/(Cnr*dxnr/(slices-1));

thisTempIncrease $=$ tempDecrease -

thisTempDecrease $=$

timeInterval*knr*((tempSlices[i])-tempSlices $[i+1]) /(C n r * d x n r /($ slices -1$))$;

\}

$\mathrm{T} 2 \mathrm{~B}=$ tempSlices[i] + thisTempIncrease-thisTempDecrease;

deltaTemp = T2B-T2A;

if $(T 2 B<\theta)$

$\mathrm{T} 2 \mathrm{~B}=\theta$;

if $(T 2 A<\theta)$

$\mathrm{T} 2 \mathrm{~A}=0$;

int multFactor $=1$;

\section{S 12}


final temperature.

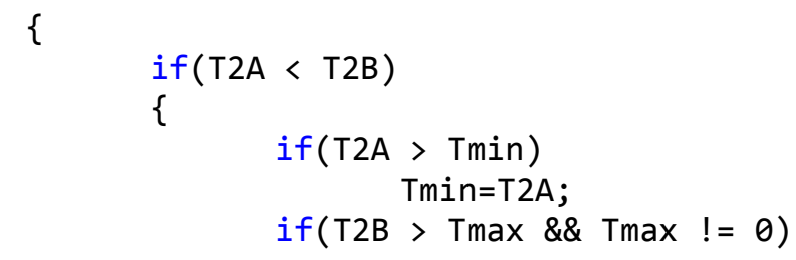


( T1))

if $((\mathrm{T} 2 \mathrm{~A}<\mathrm{Tmax} \& \& \mathrm{Tmax}>\mathrm{T} 1) \|(\mathrm{T} 2 \mathrm{~A}>\mathrm{Tmax} \& \& \mathrm{Tmax}$

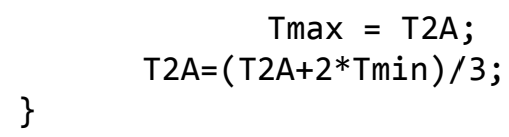




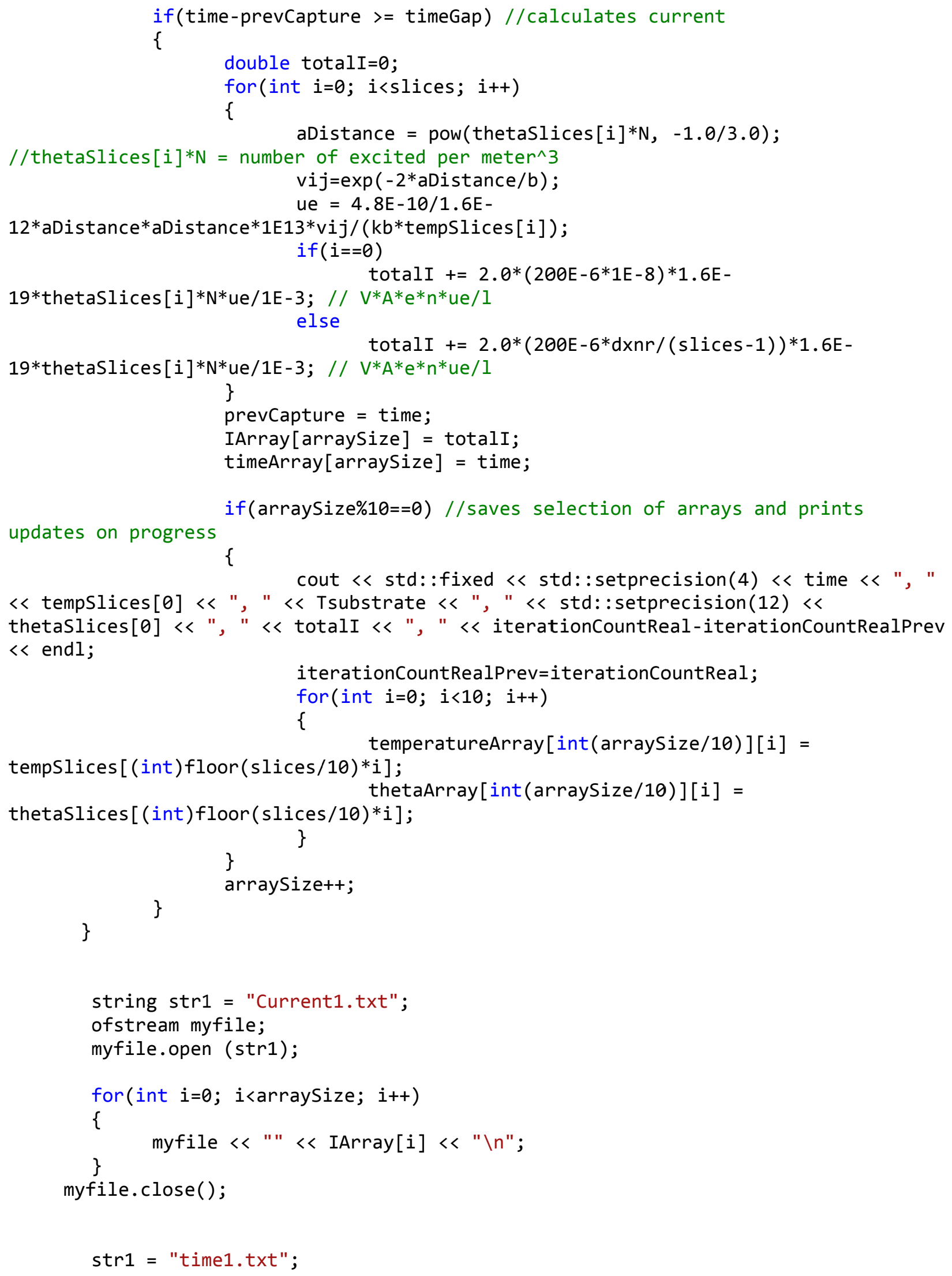




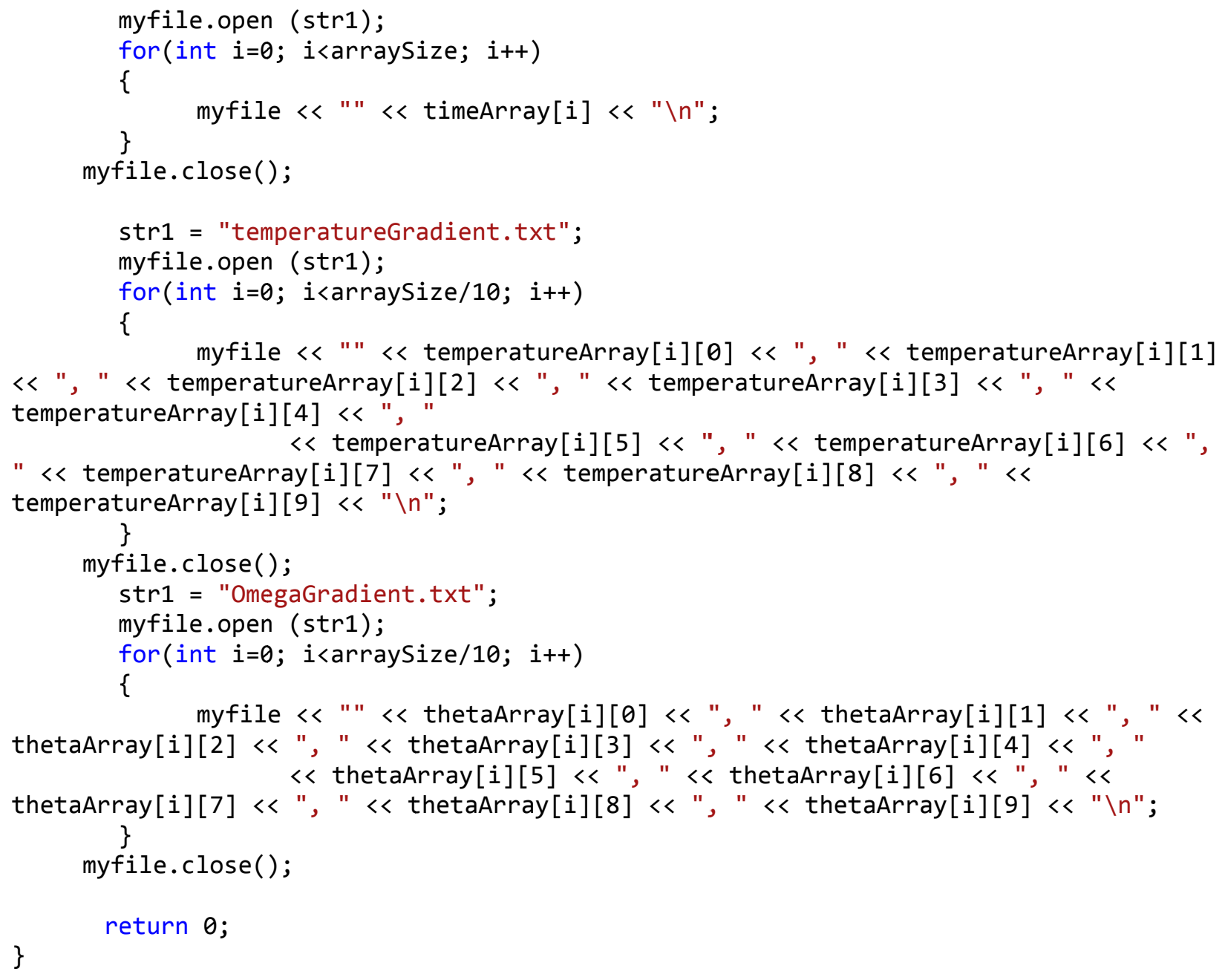

